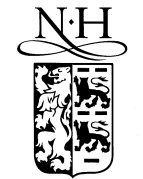

ELSEVIER

\title{
Self-referenced fibre optic intensity sensor based on a multiple beam Sagnac topology
}

\author{
J.M. Baptista ${ }^{\text {a,c, }, ~}$, J.L. Santos ${ }^{\text {b,c, * }}$, A.S. Lage ${ }^{\text {d,2 }}$ \\ ${ }^{a}$ Dep. de Engenharia Electrotécnica, Instituto Superior de Engenharia do Porto, Rua de São Tomé, 4200-072 Porto, Portugal \\ ${ }^{\mathrm{b}}$ Dep. de Física, Faculdade de Ciências, Universidade do Porto, Rua do Campo Alegre 687, 4169-007 Porto, Portugal \\ c INESC Porto, Unidade de Optoelectrónica e Sistemas Electrónicos, Rua do Campo Alegre 687, 4169-007 Porto, Portugal \\ ${ }^{\mathrm{d}}$ Dep. de Engenharia Electrotécnica e de Computadores, Faculdade de Engenharia, Universidade do Porto, Rua dos Bragas, \\ 4099 Porto Codex, Portugal
}

Received 16 March 2000; accepted 24 May 2000

\begin{abstract}
A theoretical and experimental characterisation of a self-referenced fibre optic intensity sensor based on a multiple beam Sagnac configuration is conducted. Via the definition of the measurement parameter ( $R$ parameter) sensor linearity and sensitivity are analysed. Theoretical and experimental results are compared, being considered the problem of sensor design and optimisation. (C) 2000 Elsevier Science B.V. All rights reserved.
\end{abstract}

\section{Introduction}

Optical fibre intensity-modulated sensors are very attractive since they are conceptually simple, reliable, small-sized and offer a wide range of applications at lower costs [1]. However, to ensure accurate measurements by the optical fibre intensity modulated sensors, the implementation of a reference channel is vital. Such a channel should provide

\footnotetext{
* Corresponding author. Tel.: +351-22-608-2601; fax: +35122-608-2799; e-mail: jlsantos@goe.fc.up.pt

${ }^{1}$ Tel.: +351-93-840-5238, +351-22-608-2601; fax: +351-22832-1159, +351-22-608-2799; e-mail: jmb@dee.isep.ipp.pt, jmb@goe.fc.up.pt.

${ }^{2}$ Tel.: +351-22-204-1834; fax: +351-22-200-3610; e-mail: alage@alf.fe.up.pt.
}

insensitivity to source intensity fluctuations and to variable optical transmission losses in the fibre link, couplers and connectors, which are often indistinguishable from transducer caused effects [2].

The implementation of a certain referencing technique in order to eliminate the effects of unwanted modulation depends in general on the specific sensor transduction mechanisms. There are several ways to perform a reference channel and usually all techniques that were previously reported belong to one of the following encoding mechanisms: time-of-flight difference [3], wavelength encoding [4] or frequency response [5].

The use of the interfering concept as the basis of a self-referenced intensity type sensor has already been identified in the literature, in an approach that is 
known as amplitude-phase conversion [6,7]. There, the optical power injected into the system is sinewave modulated. In the sensing head a fraction of that power is not affected by the measurand, constituting a reference signal. This, when combined with the other fraction that was intensity modulated by the measurand, gives a resulting optical power sinewave with a phase relative to the electrical signal that modulates the optical power emitted by the optical source. This phase is only dependent on the optical loss induced in the sensor head by the measurand (apart from a constant factor determined by the length of the lead/return fibre). The evaluation of this relative phase allows to obtain information about the measurand status independently of the optical power fluctuations that can occur outside the sensing head.

We have developed another frequency based approach to have a self-referenced intensity type optical fibre sensor [8,9]. It relies on the degree of constructive interference between the intensity sinewaves generated in the sensing cavity when it is illuminated by light from an optical source with its intensity modulated at different frequencies. For a fixed cavity length this degree of constructive interference is only dependent on the sinewave frequency. Therefore, when it is done the ratio of the amplitudes of the two signals, (corresponding to the interference of the waveforms arriving from the sensing cavity) obtained when the intensity of the optical source is modulated at two different frequencies, then the result only depends on the losses induced by the measurand in the sensor head, being independent on any other optical loss that can occur along the remaining optical system. In the present work this concept is developed further and the performance of the interferometric intensity sensor based on a multiple beam Sagnac sensing head topology is evaluated in detail.

We begin by characterising the sensing head transfer function. The measurement parameter is defined, allowing to analyse the sensor linearity and sensitivity. A comparison of its characteristics for two realisations of the sensing configuration is also presented. Finally, the theoretical and experimental results are compared and paths for future work are given.

\section{Sensor concept}

The proposed sensor concept is illustrated in Fig. 1. It is based on a Sagnac topology with some degree of feedback from the mirrored end face of port 4 of fibre coupler 2 . The frequency response of this structure when the input optical power from an optical source intensity is modulated at a particular frequency shows that for some frequencies the amplitude of the output optical power waveform is maximum (constructive interfering frequencies), while for other frequencies it results in a smaller value for that amplitude (the off-constructive interference frequencies). Therefore, equal intensity modulation of the light injected into this fibre structure but at different

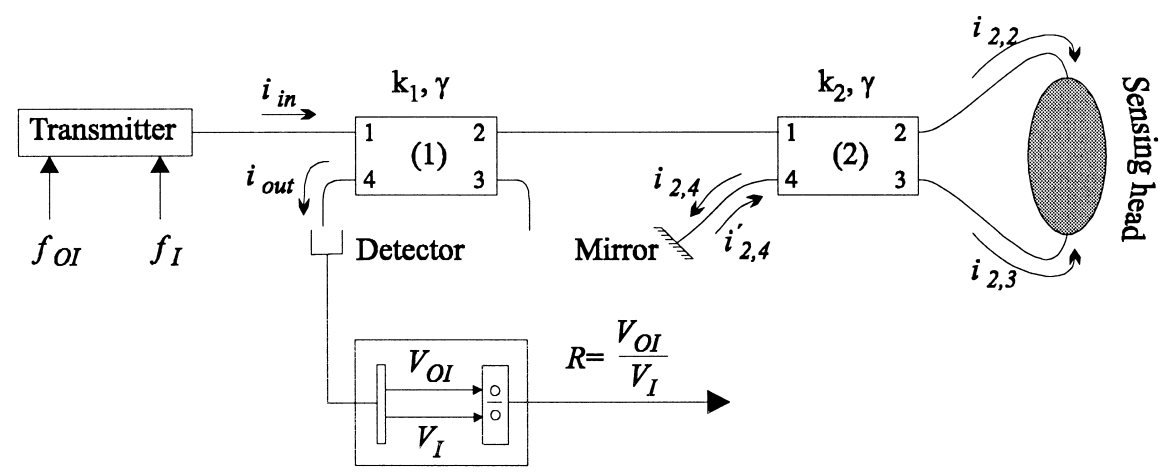

Fig. 1. Block diagram of the sensing structure based on a multiple beam Sagnac topology. $V_{\mathrm{OI}}$ and $V_{\mathrm{I}}$ are respectively voltage amplitudes proportional to the amplitude of the output optical power sinewave waveforms at an off-constructive interference frequency $\left(f_{\mathrm{OI}}\right)$ and at a constructive interference frequency $\left(f_{\mathrm{I}}\right)$. 
frequencies results in different amplitudes for the output optical power waveforms. The plot of these amplitudes versus the optical source electric modulating frequency gives the transfer function of this sensing configuration. Fig. 2 illustrates such transfer function when the Sagnac structure is subjected to losses $\left(k_{2}=0.5\right)$. This function comes from the theoretical analysis given next section and will be addressed in detail later. The parameter $g$ indicates the optical power attenuation factor externally induced in the fibre structure. For $g=1$, there is no induced optical loss and the amplitude of the modulated output light is maximum, while for $g=0$ the light is completely lost. It can be seen that when the value of the induced losses rises, in other words when $g$ gets closer to zero, the difference between the peaks and the valleys shortens until the frequency response becomes an horizontal line correspondent to no light exiting the cavity. On the other hand, when there are no external losses induced in the fibre interferometer $(g=1)$, the differences between the peaks and the valleys are at their maximum, being only dependent on the internal losses of the fibre structure (losses in fibre, splices, couplers, etc.).

As will be shown in the next section, the ratio of the value of the transfer function at an off-constructive interference frequency to its value at a constructive interference frequency depends only on the optical losses inside the fibre sensing structure (intrinsic and induced losses), being not influenced by optical power fluctuations that can occur outside the sensing head. Therefore, the modulation of that ratio pro-

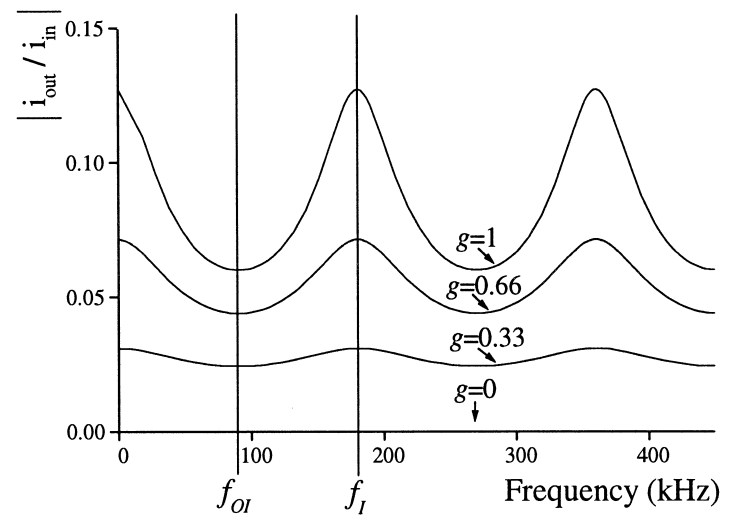

Fig. 2. Transfer function of the multiple beam Sagnac topology with the induced optical power loss factor $g$ as a parameter. vides a self-referencing scheme that makes the measurand read-out independent of possible unwanted light intensity modulation along the optical system.

\section{Theoretical analysis}

\subsection{Transfer function}

For the topology shown in Fig. 1, let the optical power into the system be:

$I_{\text {in }}=I_{\mathrm{o}}+i_{\text {in }}$

where $I_{\mathrm{o}}$ is a constant term and $i_{\text {in }}=A \cos (\omega t)$ is a phasor modulated at the angular frequency $\omega(\omega=$ $2 \pi f$ ). Neglecting the constant terms of the optical power and considering only the phasors, it turns out:

$i_{2,2}=(1-\gamma)^{2}\left(1-k_{1}\right)\left(1-k_{2}\right) i_{\text {in }}+(1-\gamma) k_{2} i_{2,4}$

$i_{2,3}=(1-\gamma)^{2}\left(1-k_{1}\right) k_{2} i_{\text {in }}+(1-\gamma)\left(1-k_{2}\right) i_{2,4}$

$i_{2,4}=(1-\gamma)\left(1-k_{2}\right) h(L) g \mathrm{e}^{-\mathrm{j} \phi} i_{2,2}$

$$
+(1-\gamma) k_{2} h(L) g \mathrm{e}^{-\mathrm{j} \phi} i_{2,3}
$$

$i_{\text {out }}=(1-\gamma)^{2} k_{1}\left(1-k_{2}\right) h(L) g \mathrm{e}^{-\mathrm{j} \phi} i_{2,3}$

$$
+(1-\gamma)^{2} k_{1} k_{2} h(L) g \mathrm{e}^{-\mathrm{j} \phi} i_{2,2}
$$

where $i_{2,4}^{\prime}=i_{2,4}$ considering a perfect mirror, $i_{x, m}$ is the optical power at port $m$ of the $x$ th coupler with coupling coefficient $k_{x},(1-\gamma)$ is the excess loss factor of the couplers, $g$ is the measurand induced loss factor and $\mathrm{e}^{-\mathrm{j} \phi}$ is the factor that represents the time delay of the optical power that travels through the fibre loop of the structure, where $\phi=2 \pi(n f L / c)$ is the static electric phase difference, being $n$ the refractive index of the fibre, $c$ the velocity of the light in vacuum and $L$ the length of the fibre loop. In (4) and (5) $h$ is the light propagation attenuation factor in fibre, which is given by $h(L)=10^{(-\alpha L / 10)}$ where $\alpha$ is the fibre propagation loss per unit length expressed in decibels per metre.

Defining the system transfer function as the module of the complex ratio $i_{\text {out }} / i_{\text {in }}$, it turns out the result illustrated in Fig. $3\left(k_{1}=0.5, \gamma=0.04, g=1\right.$, $n=1.48, L=1125 \mathrm{~m})$. For these conditions, the 


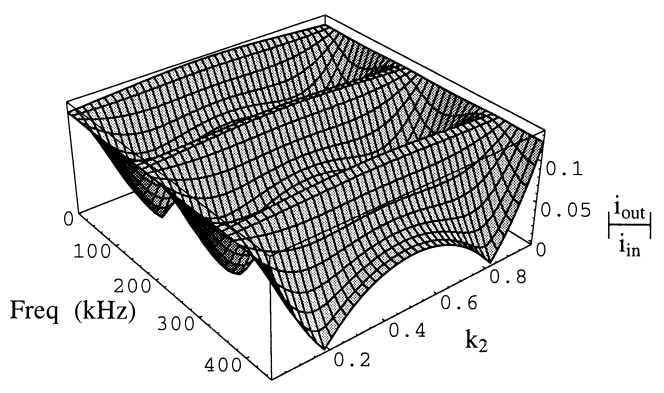

Fig. 3. Transfer function of the multiple beam Sagnac configuration versus the coupling coefficient $k_{2}$ (it is assumed that no losses are induced in the fibre structure, i.e. $g=1$ ).

maximum difference between peaks and valleys occurs for a $k_{2}=0.18$ or $k_{2}=0.82$. This difference between the peaks and valleys in the transfer function is very important because it is directly related to the sensitivity and measurement range of the sensor.

Fig. 4(a) and 4(b) show in more detail the transfer function versus the modulating frequency for the cases, $k_{2}=0.5$ and $k_{2}=0.18$, respectively. As it can be observed, these pictures are two slices of the previous graph (Fig. 3) at the points $k_{2}=0.5$ and $k_{2}=0.18$. The frequencies corresponding to the first valley and to the first constructive interference peak are $90.09 \mathrm{kHz}$ and $180.18 \mathrm{kHz}$, respectively.

To explain the operation principle of the sensing head let us consider first that the mirror, shown in Fig. 1 was not inserted in the structure. For that situation, the static electric phase difference would be null, since the optical light at coupler 2 would experience two similar paths $\left(i_{2,2}\right.$ and $\left.i_{2,3}\right)$ and consequently, the transfer function would be a horizontal line. The insertion of the mirror into the topology allows the static electric phase difference to be different from zero, since light that leaves coupler 2 by port 1 after travelling once through the fibre loop interferes with light that has travelled twice, three times or more through the fibre loop due to one or more reflections in the mirror, resulting in a multiple beam interference system. In principle, light that travels $p$ times through the fibre loop interferes with light that travels $p+q$ times in a way that depends on the loop optical length and on the modulation frequency ( $p, q$ : integers). Obviously, due to propagation losses in the fibre and due to the insertion coupler loss, the highest interference effect will occur between light that has travelled once through the fibre loop with light that has travelled twice through the same loop. Therefore, the existence of a non-zero static electric phase difference between these recirculating beams is responsible for the variation of the transfer function accordingly to the frequency value at which light is being modulated.

The values indicated above for the valley and peak frequencies are inversely proportional to the fibre length of the interferometer. In general, it is desirable to have a limited fibre length for these structures, being therefore needed a larger modulation frequency, which is not demanding in terms of system bandwidth considering the sinusoidal modulation employed does not introduce higher harmonics than the fundamental. For example, for a fibre length of $50 \mathrm{~m}$ with a fibre core refractive index of 1.48 , the first constructive interference peak would occur at a modulation frequency of $4.054 \mathrm{MHz}$. The reason

(a)

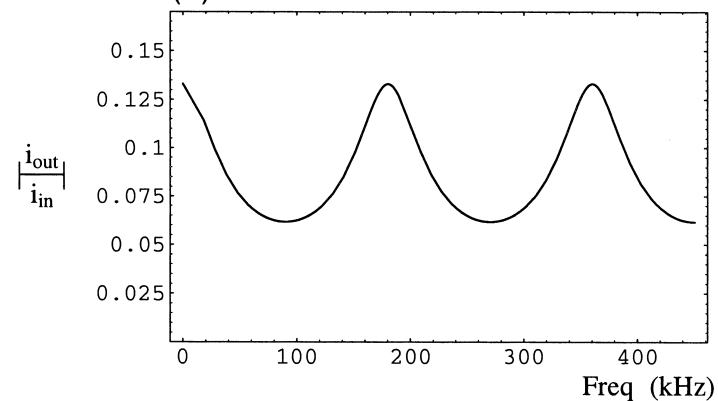

(b)

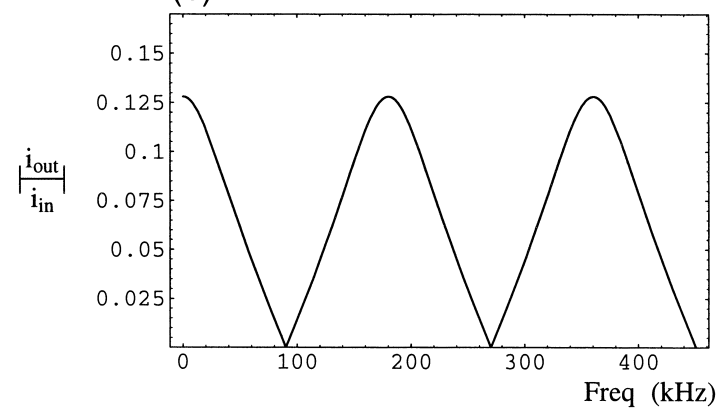

Fig. 4. Transfer function of the multiple beam Sagnac configuration for $k_{2}=0.5$ (a) and $k_{2}=0.18(\mathrm{~b})(g=1)$. 

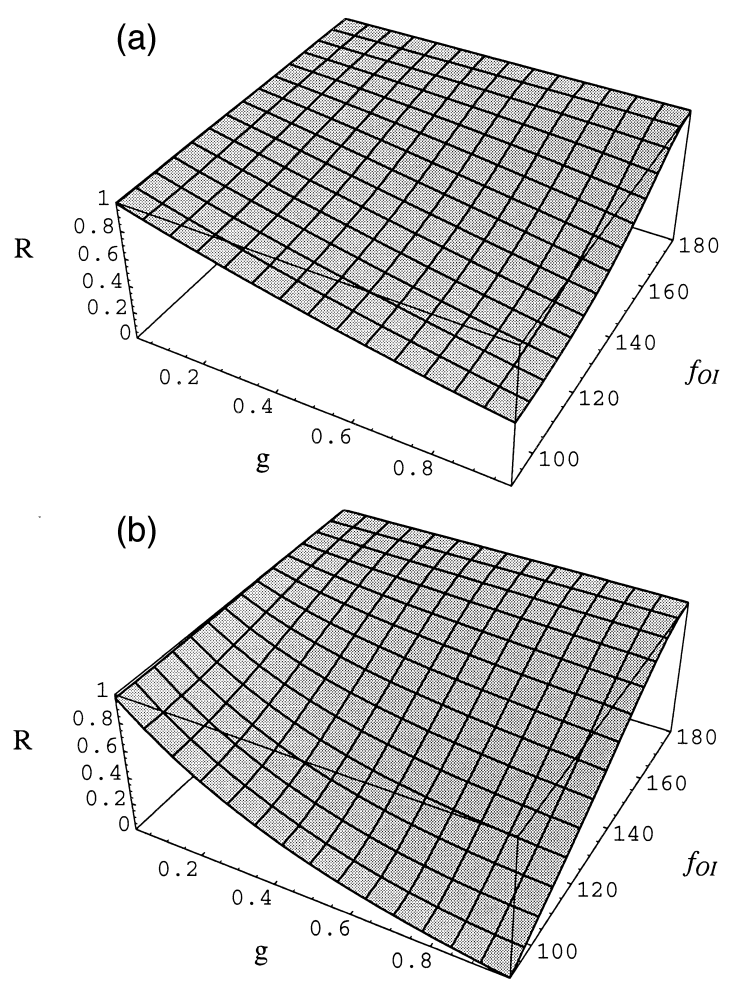

Fig. 5. Measurement parameter for the multiple beam Sagnac topology for $k_{2}=0.5$ (a) and $k_{2}=0.18$ (b) versus the induced loss factor $g$ and the off-constructive interference frequency.

of having selected the fibre length of $1125 \mathrm{~m}$ for the simulation results presented in this section is due to the fact that value was the fibre length of a fibre coil existent in the laboratory which was used in the experimental set-up.

\subsection{Measurement parameter}

In this section the behaviour of the sensing structure is further analysed. It is clearly important to know what is the best value for the off-constructive interference frequency and for the coupling coefficient of the Sagnac coupler in order to optimise the sensitivity and linearity of the sensor. The sensing operational concept is based on the definition of the following parameter, hereafter referred as the measurement parameter:

$R \equiv \frac{V_{\mathrm{OI}}}{V_{\mathrm{I}}}$ where $V_{\mathrm{OI}}$ and $V_{\mathrm{I}}$ are voltage values proportional to the amplitudes of the optical output sinewaves at an off-constructive interference and at a constructive interference frequency, respectively (Fig. 1). Fig. 5(a) and 5(b) show this parameter for the Sagnac structure when $k_{2}=0.5$ and $k_{2}=0.18$, respectively, with two variables, namely the induced optical loss factor $(g)$ and the off-constructive interference frequency $\left(f_{\mathrm{OI}}\right)$. The loss factor was varied from 0 to 1 , while the off-constructive interference frequency was changed from the value correspondent to the first valley up to the one associated with the first constructive interference peak (the other parameters had the values stated before). As it can be observed, in both cases the measurement parameter converges to unity in two regions. One of them is when $g=0$ (no light exits the sensing structure), situation in which the transfer function is constant for all frequencies, i.e., $R=1$. The other region is when the off-con-

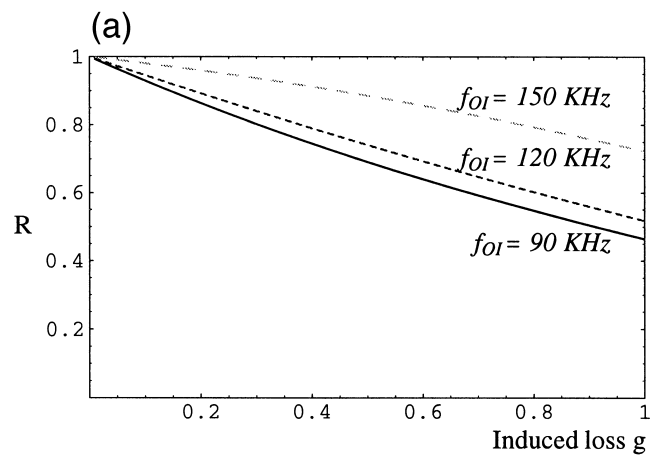

(b)

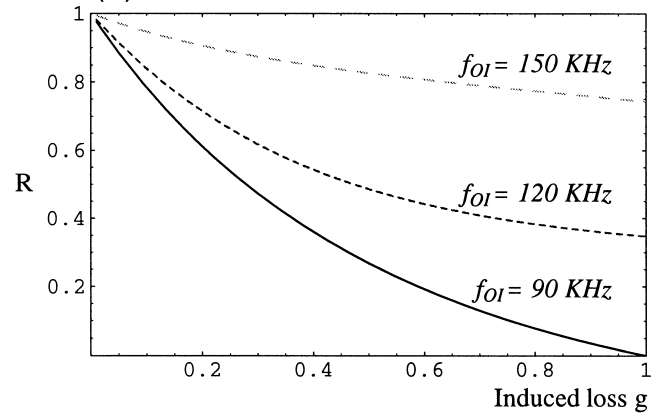

Fig. 6. Measurement parameter for the multiple beam Sagnac topology when $k_{2}=0.5$ (a) and $k_{2}=0.18$ (b) versus the induced loss factor $g$ for several values of the off-constructive interference frequencies. 
structive interference frequency approaches the constructive interference value $(180.18 \mathrm{kHz})$.

The freedom in choosing the off-constructive interference frequency allows sensor optimisation in order to reach a compromise between sensor sensitivity and linearity. The optimum value for the offconstructive interference frequency was found to be the valley frequency $(90.09 \mathrm{kHz})$. Fig. 6(a) and 6(b) show the measurement functions for several values of the off-constructive interference frequencies considering $k_{2}=0.5$ and $k_{2}=0.18$, respectively.

With respect to sensor measurement linearity the configuration with $k_{2}=0.5$ is more favourable than that associated with $k_{2}=0.18$. For $k_{2}=0.5$ the best performance occurs for $f_{\mathrm{OI}}=120 \mathrm{kHz}$, (linear correlation coefficient of 0.9984 ), while for $k_{2}=0.18$ it happens when $f_{\mathrm{OI}}=150 \mathrm{kHz}$, (linear correlation coefficient of 0.9593).

Relatively to sensor sensitivity only the configuration with $k_{2}=0.18$ and $f_{\mathrm{OI}}=90.09 \mathrm{kHz}$ is able to address the full range of $R$ values presenting in general a larger sensitivity. It is also important to state that the value found for $k_{2}(=0.18)$ that maximises the difference between the peaks and valleys of the system transfer function is not a fixed one but depends actually on the intrinsic losses of the Sagnac topology. In fact, for higher intrinsic losses than the ones considered here, values smaller than 0.18 for the Sagnac coupling coefficient are required, allow- ing the sensor to address the full range of the measurement parameter.

\section{Experiment}

To demonstrate the sensing concept described above, the experimental arrangement shown in Fig. 7 was implemented. Two sets of data were obtained, one related with $k_{2}=0.51$ and the other with $k_{2}=$ 0.17 . The coupling ratio of the input coupler was $k_{1}=0.5$ and the excess loss of the couplers were $\gamma_{1}=\gamma_{2}=0.045$, respectively. Two sinusoidal electrical signals with different frequencies were superimposed on the bias current of the light source emitting at $1300 \mathrm{~nm}$ (Edge Emitting LED, MRV MREDMP020-1). Multimode fibre and couplers $(50 / 125 \mu \mathrm{m})$ were used. The length of the fibre roll used was approximately $1125 \mathrm{~m}$. In combination with the fibre core refractive index, this corresponds to a frequency for the first constructive interference peak of $180.18 \mathrm{kHz}$. A multimode fibre attenuator (JDS Fitel) was used to induce power loss in the Sagnac structure. The fibre end reflectivity of port 4 of coupler 2 was approximately $100 \%$ by utilisation of an appropriated silvering technique. A mode scrambler was inserted in order to equalise the modal

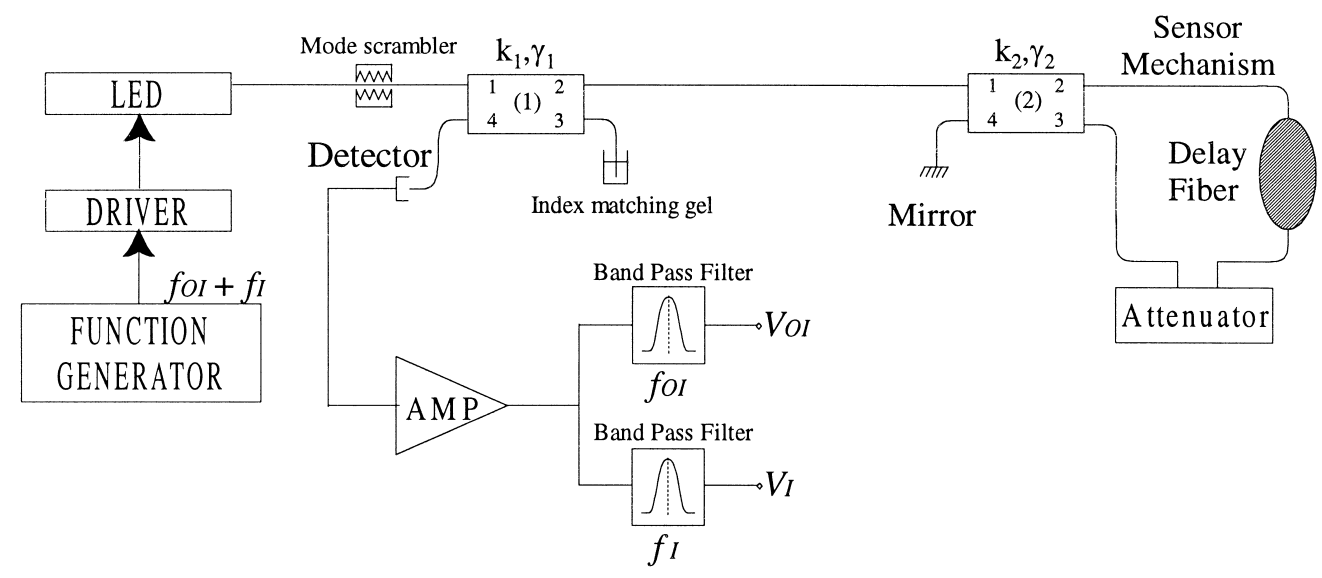

Fig. 7. Experimental arrangement. 
distribution in the input fibre. To observe the sensor transfer function one of the electrical signals was swept. In order to obtain the $R$ parameter two band pass filters centred at the frequencies of the sinewaves that modulate the injection current of the optical source were used, being determined the rms values of the correspondent output voltage signals which are proportional to their amplitudes.

\section{Results}

Fig. 8(a) and 8(b) show the experimentally determined transfer functions for the multiple beam Sagnac configuration when $k_{2}=0.51$ and $k_{2}=0.17$, respectively. They clearly show the importance of the choice of the coupling coefficient value of the Sagnac coupler. For these two cases, Fig. 9(a) and

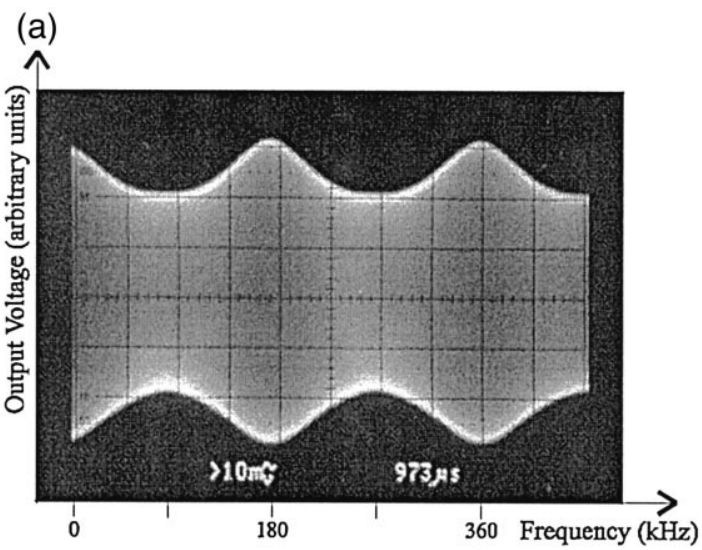

(b)

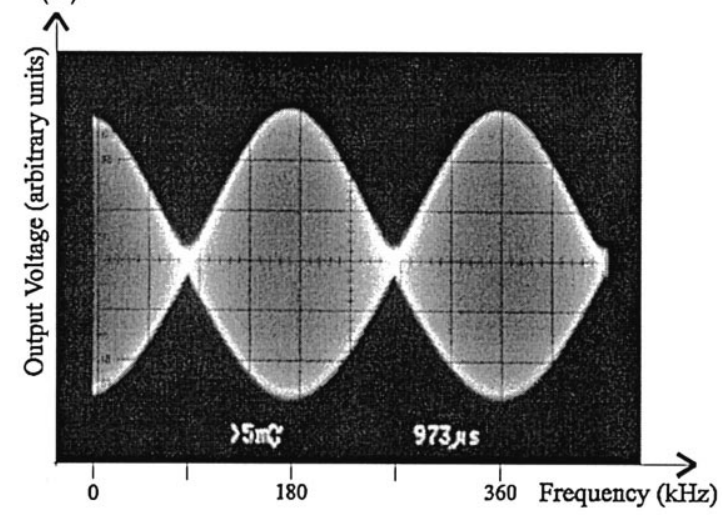

Fig. 8. Experimental transfer functions for the multiple beam Sagnac topology when $k_{2}=0.51$ (a) and $k_{2}=0.17$ (b). (a)

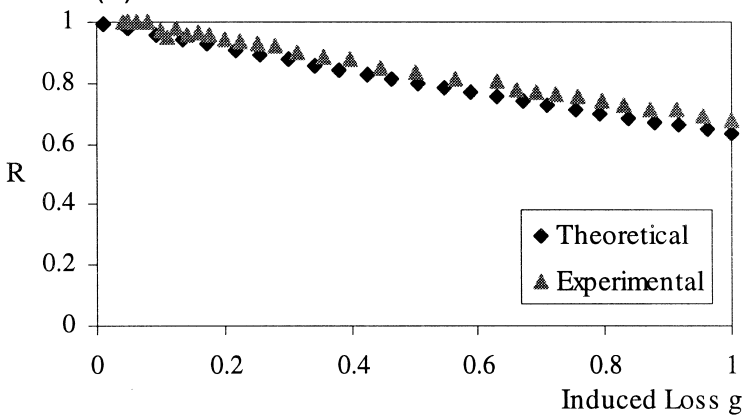

(b)

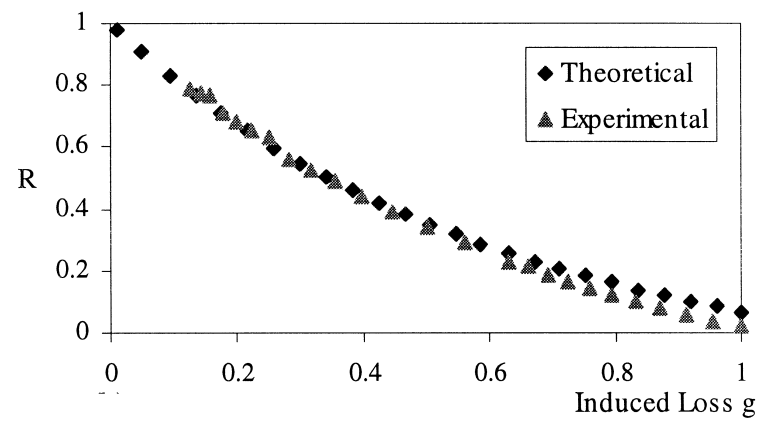

Fig. 9. Experimental and theoretical results for the measurement parameter when $k_{2}=0.51$ (a) and $k_{2}=0.17$ (b).

9(b) plot the results obtained for the $R$ parameter for the two cases, when the off-constructive interference frequency was set to the one relative to the first valley $(90.09 \mathrm{kHz})$.

\section{Discussion}

Comparing the theoretical with the experimental results, it can be stated that they are in fairly good agreement. The sensing structure reveals good sensitivity for $k_{2}$ around 0.2 , being the optimum value dependent on the intrinsic losses of the configuration. In what concerns the measurement range, it can be increased if the intrinsic optical losses in the sensing structures are reduced (losses in couplers, splices, fibre, etc). A feature also evident from data of Fig. 9 is that the sensor linearity obtainable with $k_{2}=0.5$ is better than the one which results when $k_{2} \approx 0.2$. However, further processing can always improve this characteristic. 
A sensing structure with high sensitivity is particularly important for monitoring dynamic parameters (e.g. vibration), which do not normally have large amplitudes and, consequently, originate correspondingly small induced optical losses. It must be emphasised that the sensitivity to a given measurand depends not only on the primary sensitivity of the sensing structure but also on the interface between the measurand action and the optical loss induced in the sensor head. This is clearly an important issue, which needs to be addressed in each particular case.

The measured system resolution was found to be $-14.5 \mathrm{~dB} / \sqrt{\mathrm{Hz}}$. It is important to keep in mind that the sensing concept described in this work is particularly favourable in what concerns the minimisation of system noise. This happens because what is monitored is the amplitude of two sinewaves, i.e. the detection bandwidth can be made as narrow as practically feasible, with the consequent decrease of the system noise level.

The sensing configuration described in this work is a versatile structure for monitoring of static and dynamic physical parameters, requiring a small number of optical components and simple signal processing. Of relevant importance is the underlying concept of self-referencing which naturally solves the problem related to optical power fluctuations and others that can occur along the system.
In conclusion, in this paper we have described a concept of self-referenced intensity based optical fibre sensor and applied it to a multiple beam Sagnac configuration. Theoretical and experimental results were presented. The model developed proved to be effective in describing the sensor properties, which validates its utilisation for sensor design and optimisation.

\section{References}

[1] J. Dakin, B. Culshaw (Eds.), Optical Fibre Sensors: Applications, Analysis and Future Trends, Artech House, London, 1997.

[2] G. Murtaza, J.M. Senior, Int. J. Optoelectron. 9 (4) (1994) 339.

[3] G. Adamovsky, Appl. Opt. 27 (7) (1988) 1313.

[4] G. Murtaza, J.M. Senior, IEEE Photon. Technol. Lett. 6 (8) (1994) 1020

[5] R.I. MacDonald, R. Nychka, Electron. Lett. 27 (23) (1991) 2194.

[6] D.N. Davies, J. Chaimowicz, G. Economou, J. Foley, Proc. 2nd Optical Fibre Sensors, 1984, p. 387.

[7] P. Sixt, G. Kotrotsios, L. Falco, O. Parriaux, J. Lightwave Technol. 4 (7) (1986) 926.

[8] J.M. Baptista, P.M. Cavaleiro, J.L. Santos, Int. J. Optoelectron. 10 (2) (1995) 105

[9] J.M. Baptista, P.M. Cavaleiro, J.L. Santos, Rev. Sci. Instrum. 67 (11) (1996) 3788. 